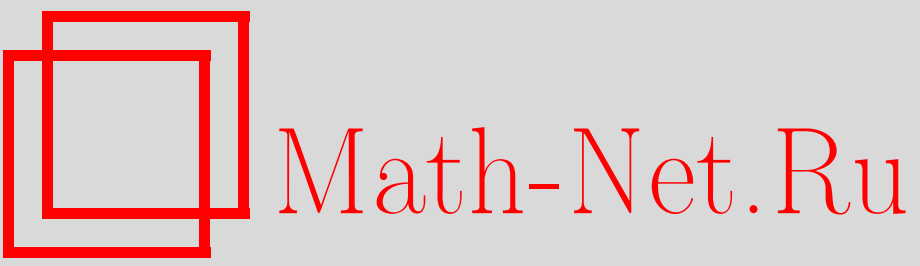

С. В. Людковский, Неархимедовы полиэдральные разложения ультраравномерных пространств, УМН, 1999, том 54, выпуск 5, 163-164

DOI: https://doi.org/10.4213/rm211

Использование Общероссийского математического портала Math-Net.Ru подразумевает, что вы прочитали и согласны с пользовательским соглашением

http://www . mathnet.ru/rus/agreement

Параметры загрузки:

IP: 34.229 .45 .116

26 апреля 2023 г., 06:32:21 


\title{
НЕАРХИМЕДОВЫ ПОЛИЭДРАЛЬНЫЕ РАЗЛОЖЕНИЯ УЛЬТРАРАВНОМЕРНЫХ ПРОСТРАНСТВ
}

\author{
С.В. Людковский
}

Разложению топологических и равномерных пространств в виде пределов обратных спектров были посвящены работы [1], [2]. Данная работа посвящена исследованию вопроса о спектральной разложимости ультраравномерных пространств с полиэдрами над неархимедовыми локальными полями $L \supset \mathbb{Q}_{p}$ (см. о таких полях [3]).

Ультраметрическим пространством $(X, \rho)$ назьвается множество $X$ с метрикой $\rho$, удовлетворяющей неравенству $\rho(x, y) \leqslant \max \{\rho(x, z) ; \rho(z, y)\} \forall x, y$ и $z \in X$. Ультраравномерным назьвается равномерное пространство $(X, \mathrm{~K})$ с ультраравномерностью $\mathrm{K}$, удовлетворяющей условию $|x-z|<V^{\prime}$, если $|y-z|<V^{\prime}$ и $|y-x|<V$, где $V \subset V^{\prime} \in \mathrm{K} \forall x, y$ и $z \in X[3]$.

ОПредЕлЕниЕ 1. (1) Полиэдром $P$ в $c_{0}(L, n)$ назовем дизъюнктное объединение симплексов $s_{j}, P=\bigsqcup_{j \in F} s_{j}, F$ - множество, $s_{j}=B\left(c_{0}\left(L, m_{j}\right), x, r\right)=x+\pi_{L}^{a} B\left(c_{0}\left(L, m_{j}\right), 0,1\right)$, где $m_{j} \leqslant n, a \in \mathbb{Z}, B\left(c_{0}(L, m), x, r\right):=\left\{y \in c_{0}(L, m):\|x-y\| \leqslant r\right\} ; c_{0}\left(L, m_{j}\right) \hookrightarrow c_{0}(L, n) ;$ $c_{0}(L, w):=\left\{x: x=\left(x_{j}: j \in \alpha\right), x_{j} \in L, \forall \varepsilon>0 \operatorname{card}\left(j:\left|x_{j}\right|_{L}>\varepsilon\right)<\aleph_{0}\right\}$ - это банахово пространство над полем $L \mathrm{c}\|x\|:=\sup _{j}\left|x_{j}\right|, \alpha-$ ординал, $\operatorname{card}(\alpha)=w ; \operatorname{dim}\left(L, s_{j}\right):=m_{j}$. Для каждого $L$ зафиксируем $\pi_{L} \in L$ такие, что $B\left(L, 0,1^{-}\right):=\{y \in L:|x|<1\}=\pi_{L} B(L, 0,1)$, и такие аффинные преобразования. Полиэдр назьвается равномерным, если (i) $\sup \left\{\operatorname{diam}\left(s_{j}\right)\right.$ : $j \in F\}<\infty$ и (ii) $\inf \left\{\operatorname{dist}\left(s_{j}, s_{i}\right): i \neq j\right\}>0$. Введем $p^{j}$-подразделение симплексов (полиэдров) для $j \in \mathbb{N}$, т.е. разбиение каждого симплекса $B\left(c_{0}(L, m), x, r\right)$ в дизъюнктное объединение симплексов с радиусами, равными $r p^{-j}$. Размерностью полиэдра $P$ назовем $\operatorname{dim}(L, P):=$ $\sup \left\{\operatorname{dim}\left(L, s_{j}\right): j \in F\right\}$. Полиэдр $P$ над $L$ называется локально конечномерным, если все симплексы $s$ в $P$ конечномерны над $L$. Для симплекса $s=B\left(c_{0}(L, m), x, r\right) L$-границей $\partial s$ назовем объединение всех его граней $q$ коразмерности 1 над $L$ в $c_{0}(L, m)=: X$, т.е. $q=e+B^{\prime}$, где $B^{\prime}-$ шар в $c_{0}(L, \operatorname{card}(E))=: Y, \operatorname{card}(A)=m, A \backslash E-$ одноэлементное множество, $E \subset A$, $X \ominus Y=L, Y \hookrightarrow X$. Для полиэдра $P \quad L$-границей назовем $\partial P:=\bigcup_{j \in F} \partial s_{j}$. Вершинами симплекса $s=B\left(c_{0}(L, m), 0,1\right)$ назовем $\left\{x=\left(x_{j}\right) \in c_{0}(L, m): x_{j}=0\right.$ или $x_{j}=1$ $\forall j \in A\}$. Для любого $E \subset A$ с $E \neq A$ и вершины $e$ гранью симплекса $s$ назовем подмножество $e+B\left(c_{0}(L, \operatorname{card}(E)), 0,1\right) \subset s$. Для произвольного симплекса грани и вершины определяются с помощью аффинного преобразования как образы граней и вершин единичного симплекса $B\left(c_{0}(L, m), 0,1\right)$.

(2) Непрерывное отображение $f$ множества $M \subset c_{0}(L, m)$ в полиэдр $P$ назовем существенным, если не существует непрерьвного $g: M \rightarrow P$, удовлетворяющего условиям (i)-(iii):

(i) $g(M) \not \supset P$;

(ii) имеется $M_{0} \subset M, M_{0} \neq M$ с $f(M) \cap \partial P=f\left(M_{0}\right)=g\left(M_{0}\right) \subset \partial P$ и $\left.f\right|_{M_{0}}=\left.g\right|_{M_{0}}$;

(iii) если $f$ линейно на $[x, y]:=\{t x+(1-t) y \mid t \in B(L, 0,1)\} \subset M$, то $g$ тоже линейно на $[x, y]$, если $f(x) \neq f(y)$, то $g(x) \neq g(y)$.

(3) Пусть $f: M \rightarrow P$ и $g: M \rightarrow P$ - непрерьвные отображения, где $M$ - множество, а $P$ полиэдр, тогда $g$ называется допустимой модификацией $f$, если выполнены условия (i)-(iii):

(i) из $a \in M$ и $f(a) \in s$ следует, что $g(a) \in s$, где $s-$ симплекс из $P$;

(ii) если $x, y \in M,[x, y] \subset M$ и $f:[x, y] \rightarrow P$ линейно, то $g:[x, y] \rightarrow P$ тоже линейно; если $f(x) \neq f(y)$, то $g(x) \neq g(y)$

(iii) $f(\partial M)=g(\partial M)$.

(4) Пусть $X=\lim \left\{X_{i}, f_{j}^{i}, E\right\}$ - разложение $X$ в предел обратного спектра полиэдров $X_{i}$ над $L$. Оно называется (а) неприводимым, если для любого открытого $V \subset X$ существует конфинальное подмножество $E(V) \subset E$ такое, что $\left\{X_{i}, f_{j}^{i}, E(V)\right\}$ - неприводимое полиэдральное представление пространства $V$, т.е. $f_{j}^{i}: X_{i} \rightarrow X_{j}$-неприводимы и сюръективны $\forall i \geqslant j \in E(V)$. 
Теорема 2. Пусть $(X, \mathrm{~K})$ - полное ультраравномерное пространство. Тогда существует неприводимое нормальное разложение $(X, \mathrm{~K})$ в предел обратного спектра $S=\left\{P_{i}, f_{j}^{i}, E\right\}$ равномерных полиэдров $P$ над $L$, причем $\lim S$ равномерно изоморфен $(X, \mathrm{~K}) ;$ в частности, для ультраметрического $(X, \rho)$ спектр $S$ является обратной последовательностью.

ОпредЕлЕниЕ 3 . Собственное $p^{j}$-подразделение комплекса $K^{a}$ над $L$ назьвается нормальным. Наименьший открыто-замкнутый подкомплекс комплекса $K^{a}$, содержащий $X \subset K^{a}$, называется полиэдральной окрестностью или открыто-замкнутой звездой множества $X$ относительно $K^{a}$ и обозначается $K^{a} X$. Для комплексов $K_{1}^{a}$ и $K_{2}^{a}$ их соединение $K_{1}^{a} \circ K_{2}^{a}$ определено в $c_{0}\left(L, w\left(K_{1}^{a}\right)\right) \oplus c_{0}\left(L, w\left(K_{2}^{a}\right)\right) \oplus L$ аналогично классическому случаю с заменой $\{x: 0 \leqslant x \leqslant 1\}=$ $[0,1] \subset \mathbb{R}$ на $B(L, 0,1)=[0,1] \subset L$, где $K_{j}^{a} \hookrightarrow c_{0}\left(L, w\left(K_{j}^{a}\right)\right)$. Отображение $h: K \rightarrow N$ называется линейным относительно комплексов $K^{a}$ и $N^{a}$, если оно симплициально для соответствующих полиэдров. Комплекс $K^{a}$ назовем равномерным, если соответствующий ему полиэдр над $L$ является равномерным. Здесь $\operatorname{dim}\left(L, K^{a}\right)$ может быть бесконечной. Абсолютный полиэдральный спектр называется равномерным сверху, если полиэдры $P$ удовлетворяют условию (i) из определения 1 (1).

ТЕОрема 4. Каждое ультраравномерное пространство $(X, \mathrm{~K})$ имеет неприводимое абсолютное (нормальное и равномерное сверху) полиэдральное представление $\mathrm{T}:=$ $\left\{X_{\beta}, f_{\alpha}^{\beta}, \mathrm{U}\right\}$ над L, то есть для любого $Y \subset(X, \mathrm{~K})$ существует направленное подмножество $\mathrm{U}(Y) \subset \mathrm{U}$ такое, что $Y$ гомеоморфно (равномерно изоморфно соответственно) $\lim$ Т. Более того, в качестве абсолютного полиэдрального представления (не обязательно равномерного) могут быть выбраны локально конечномерные $X_{\beta}$ над $L$.

ТЕОРема 5. Ультраравномерное пространство $(X, \mathrm{~K})$ гомеоморфно $\lim S$ с вещественно полным.м конечномерными над $L$ полиэдрами $P_{i}$ и неприводимым нормальным обратным спектром $S=\left\{P_{i}, f_{j}^{i}, F\right\}$ тогда и только тогда, когда $X$ вещественно полHo.

Доказательства этих теорем даны в [4].

\section{СПИСОК ЛИТЕРАТУРЫ}

[1] Isbell J. R. // Indag. Math. Ser. A. 1961. V. 23. №1. Р. 242-248. [2] Козловский И. М. // Труды MMO. 1979. T. 40. C. 83-119. [3] van Rooij A. C. M. Non-Archimedean Functional Analysis. New York: Dekker, 1978. [4] Ludkovsky S. V. // Preprint IHES/M/97/90. Inst. Haut. Étud. Sci, 1997. 